\title{
Effect of hesperetin derivatives on the development of selenite-induced cataracts in rats
}

\author{
YOSUKE NAKAZAWA ${ }^{1}$, MARTIN PAUZE $^{1}$, KAZUYA FUKUYAMA $^{1}$, NORIAKI NAGAI ${ }^{2}$, \\ MEGUMI FUNAKOSHI-TAGO ${ }^{1}$, TAKESHI SUGAI ${ }^{1}$ and HIROOMI TAMURA ${ }^{1}$
}

\author{
${ }^{1}$ Faculty of Pharmacy, Keio University, Tokyo 105-8512; ${ }^{2}$ Faculty of Pharmacy, Kindai University, Osaka 577-8502, Japan
}

Received January 17, 2018; Accepted May 15, 2018

DOI: $10.3892 / \mathrm{mmr} .2018 .9045$

\begin{abstract}
Cataracts are a major cause of blindness worldwide. As anti-cataract pharmaceutical therapies require long-term treatment, identifying anti-cataract compounds that are ubiquitous in the human diet, have no adverse effects and are affordable, is of paramount importance. The present study focused on hesperetin and its derived compounds, hesperetin stearic acid ester (Hes-S) and hesperetin oleic acid ester (Hes-O), in order to investigate their therapeutic potential to treat cataracts in a selenite animal model. Thirteen-day-old Sprague Dawley rats were divided into 12 groups. Animals in groups 1 and 7 were subcutaneously injected with vehicle, those in groups 2 and 8 were administered hesperetin, those in groups 3 and 9 received stearic acid, those in groups 4 and 10 were injected with oleic acid, those in groups 5 and 11 were administered Hes-S, and those in groups 6 and 12 received Hes-O $(10 \mathrm{nmol} / \mathrm{kg}$ body weight on days 0,1 and 2). Animals in groups 7 to 12 were treated with sodium selenite $(20 \mu \mathrm{mol} / \mathrm{kg}$ body weight given $4 \mathrm{~h}$ following the test compound treatment on day 0 ) to induce cataract. On day 6, rats had less severe central opacities and lower stage cataracts than rats in the selenite treatment-only control groups. The levels of glutathione (GSH) and ascorbic acid (AsA) in lenses with selenite-induced cataracts declined to one-third of that of controls, and the reduction in GSH and AsA levels was rescued following hesperetin, Hes-S or Hes-O treatment, with concentrations remaining to $70-80 \%$ of that of controls. However, there were no changes in the plasma levels of GSH and AsA following treatments. Administration of either hesperetin or hesperetin-derived compounds prevented the
\end{abstract}

Correspondence to: Dr Yosuke Nakazawa, Faculty of Pharmacy, Keio University, 1-5-30 Shibako-en, Minato-ku, Tokyo 105-8512, Japan

E-mail: nakazawa-ys@pha.keio.ac.jp

Abbreviations: GSH, glutathione; AsA, ascorbic acid; DTNB, dithionitrobenzene; DCPIP, 2,6-dichlorophenol-indophenol; ALDH, aldehyde dehydrogenase; SD, Sprague Dawley; Se, selenite

Key words: anti-cataract, hesperetin, hesperetin fatty acid ester, glutathione, ascorbic acid, chaperone activity reduction of chaperone activity in the lens, and rats treated with Hes-S or Hes-O treatment had significantly greater chaperone activity than hesperetin-treated rats. Collectively, these results suggested that hesperetin and hesperetin-derived compounds may be novel drug compounds that have the potential to prevent or delay the onset of cataracts.

\section{Introduction}

Cataracts refers to a clouding of the lens of the eye, which affect vision and remain a major cause of blindness in the world $(1,2)$. According to the World Health Organization (WHO), cataracts account for approximately $51 \%$ of global blindness. Therefore, cataracts are a critical public health and social problem worldwide. At present, it is impossible to entirely prevent cataract formation, and cataract surgery remains the most common method of treatment. In order to decrease the burden of surgery in older adults, it is of great interest to establish alternative therapies to delay or prevent the development of cataracts.

Lenses that are chronically exposed to ultraviolet (UV) radiations generate reactive oxygen species (ROS) and oxidative modifications (3) and have high levels of reduced glutathione (GSH) and ascorbic acid (AsA) to maintain a constant redox state, protecting against oxidative stress and preserving lens transparency. Consequently, the levels of these compounds in the lens are frequently used as markers of cataract formation or development in both human and animal models (4-7). The lens also uses chaperone activity to maintain its transparency. $\alpha$-Crystallin, which constitutes up to $30 \%$ of total water-soluble proteins in the lens, acts as a molecular chaperone. Molecular chaperone activity plays an important role in in vivo due to the longevity and negligible turnover of lens proteins. Furthermore, it is well-known that lens proteins in cataracts have weaker chaperone activity than those in the non-disease state. Therefore, we measured the effect of hesperetin and hesperetin derivatives on the changes in chaperone activity in lenses with cataracts.

We have previously reported that hesperetin, one of the natural flavonoids in orange rinds, could delay cataract onset as assessed by observing cataract grade and measuring lens GSH and AsA levels. We also showed that treatment with hesperetin prevented down-regulation of chaperone activity in the lens $(8,9)$. In the current study, we assessed the therapeutic 
ability of hesperetin derivatives to produce strong-acting anti-cataract activity using an Se-induced cataract model, a well-established rodent model used for screening potential anti-cataract molecules.

In this study, we determined whether anti-cataract properties of these derivatives could be altered by linking fatty acids. Either hesperetin or hesperetin derivatives were administered to rats with Se-induced cataracts in order to assess the anti-cataract effect of these compounds.

\section{Materials and methods}

Materials. Sodium selenite $\left(\mathrm{Na}_{2} \mathrm{SeO}_{3}\right.$ : Se), hesperetin, isoflurane, GSH, dithionitrobenzene (DTNB), AsA, metaphosphoric acid, and 2-vinylpyridine were purchased from Wako Pure Chemical Industries, Ltd. (Osaka, Japan). Stearic acid and oleic acid were purchased from Tokyo Chemical Industry Co., Ltd. (Tokyo, Japan). Trichloroacetic acid was purchased from Nacalai Tesque Inc. (Kyoto, Japan). 2,6-Dichlorophenol-indophenol (DCPIP) was purchased from Merck KGaA, (Darmstadt, Germany). Sprague Dawley (SD) rats were obtained from Sankyo Labo Service Corporation (Tokyo, Japan).

From hesperetin (Hes, Fig. 1A), we synthesized hesperetin stearic acid ester (Hes-S, Fig. 1B) and oleic acid ester (Hes-O, Fig. 1C) according to a previously reported procedure (10), and purified materials were used for the anti-cataract experiments.

Animals. SD rats were housed in temperature-controlled $\left(25^{\circ} \mathrm{C} \pm 5^{\circ} \mathrm{C}\right)$ and light-controlled rooms $(12 \mathrm{~h}$ cycle of light and dark). Animals were fed balanced rat chow (CE-2; Clea Japan, Inc., Tokyo, Japan) and provided water ad libitum. Keio University Animal Research Committee (Tokyo, Japan) approved all of the animal procedures that were performed in the present study [11014-(4)]. Rats were euthanized with isoflurane (5\%, inhalation). Blood samples were immediately collected form the vena cava; as much blood as possible was obtained from each rat for subsequent measurements. All of the animals in this work were treated according to the National Institutes of Health (NIH) guide for the care and use of laboratory animals.

Se-induced cataracts and hesperetin treatment. A total of 168 female rats that were 13 days old were randomized into 12 groups: Group 1, Control group (G1); Group 2, Treatment with hesperetin (G2); Group 3, Treatment with stearic acid (G3); Group 4, Treatment with oleic acid (G4); Group 5, Treatment with Hes-S (G5); Group 6, Treatment with Hes-O (G6); Group 7, Treatment with Se (G7); Group 8, Treatment with hesperetin and Se (G8); Group 9, Treatment with stearic acid and Se (G9); Group 10, Treatment with oleic acid and Se (G10); Group 11, Treatment with Hes-S and Se (G11); and Group 12, Treatment with Hes-O and Se (G12). (Table I; $n=6$ or 8 per group in each experiment).

Either test compound or vehicle was administered to each group of rats as described in Table I. Rats in groups G1-G6 were subcutaneously injected with phosphate-buffered saline (PBS) and those in G7-G12 were subcutaneously injected with Se at $20 \mu \mathrm{mol} / \mathrm{kg}$ body weight. PBS or Se was injected into 13-day-old rats (day 0) $4 \mathrm{~h}$ after administration of test compound. Hes, stearic acid, oleic acid, Hes-S, or Hes-O

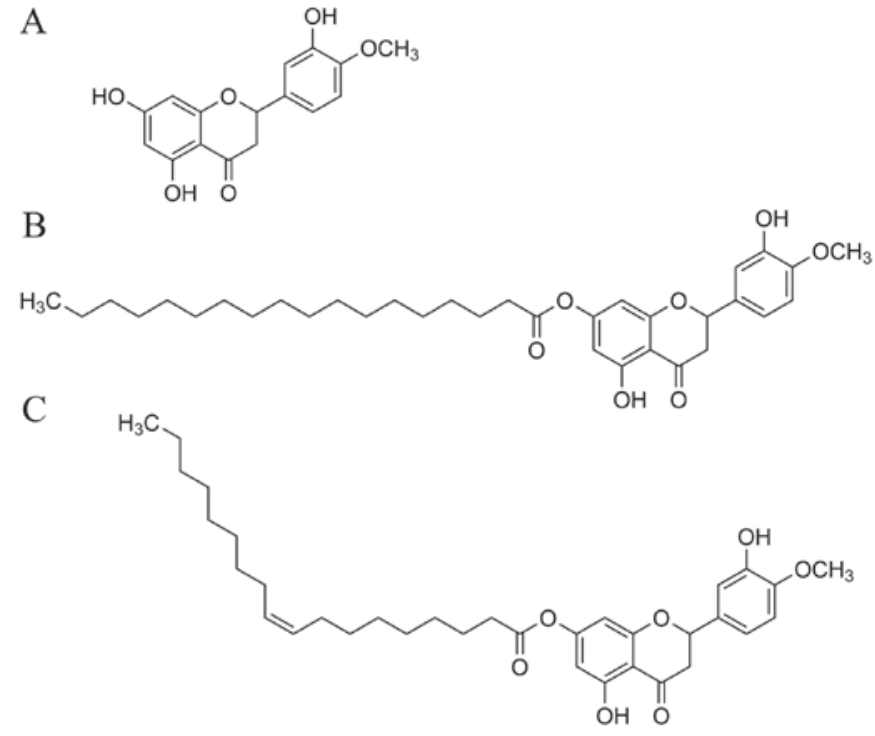

Figure 1. Structural formulas of hesperetin and hesperetin derivatives used in the present study. Structural formulas of (A) hesperetin, (B) hesperetin stearic acid ester and (C) hesperetin oleic acid ester.

was dissolved in 7\% ethanol and $93 \%$ olive oil solution and administered on Days 0, 1 and 2 subcutaneously at $10 \mathrm{nmol} / \mathrm{kg}$ body weight per day. The doses of hesperetin and its derivatives were decided according to our previous reports $(8,9)$. On day 6 , when the rats were 19 days old, following euthanization, enucleated eyes were analyzed for levels of GSH and AsA, and lens chaperone activities were determined. Plasma samples were separated by centrifugation of whole blood with heparin; plasma was stored at $-80^{\circ} \mathrm{C}$ before analysis.

Cataract classification. Cataract classifications were defined as previously described (11). Briefly, cataract stage 1 was defined as $<5 \%$ opacity in the lens, stage 2 was defined as $5-20 \%$ opacity, stage 3 was defined as $20-40 \%$ opacity, stage 4 was defined as $40-60 \%$ opacity, stage 5 was defined as $60-80 \%$ opacity, and stage 6 was defined as $>80 \%$ opacity. Following lens observation, rats were euthanized by isoflurane inhalation and lenses were recovered for further analyses.

Measurement of GSH. The level of lens GSH was determined according to a method previously described by Sedlak \& Lindsay, with minor modifications (12). Briefly, lenses were homogenized in $0.1 \mathrm{M}$ sodium phosphate buffer ( $\mathrm{pH}$ 8.0) and centrifuged. The water-soluble fraction was deproteinized using trichloroacetic acid and centrifuged to remove the proteins. The supernatant was diluted with sodium phosphate buffer according to the wet lens weight ( $1 \mathrm{mg}$ lens weight $/ \mathrm{ml})$. The sample was divided into two tubes: one tube contained $10 \mathrm{mM}$ 2-vinylpyridine to sequester GSH for measuring oxidative GSH, and the other tube contained the same volume of sodium phosphate buffer to measure the total GSH content. Both tubes were incubated for $1 \mathrm{~h}$ at room temperature in a fume hood. After incubation, the excess 2-vinylpyridine was neutralized with triethanolamine. DTNB was then added to both tubes, and the mixture was incubated for $30 \mathrm{~min}$ at room temperature. Absorbance at $412 \mathrm{~nm}$ was then measured in an Infinite M200PRO microplate reader (Tecan Ltd., Männedorf, 
Table I. Experimental groups used in the present study.

\begin{tabular}{llll}
\hline Group & Challenge & Test compound & Administration route \\
\hline Group 1 & PBS & Vehicle & S.C. \\
Group 2 & PBS & Hes & S.C. \\
Group 3 & PBS & Stealic acid & S.C. \\
Group 4 & PBS & Oleic acid & S.C. \\
Group 5 & PBS & Hes-S & S.C. \\
Group 6 & PBS & Hes-O & S.C. \\
Group 7 & Se & Vehicle & S.C. \\
Group 8 & Se & Hes & S.C. \\
Group 9 & Se & Stealic acid & S.C. \\
Group 10 & Se & Oleic acid & S.C. \\
Group 11 & Se & Hes-S & S.C. \\
Group 12 & Se & Hes-O & S.C.
\end{tabular}

Sprague Dawley 13-day-old rats were randomized into 12 groups. Rats in groups 1-6 were injected with PBS. Rats in groups 7-12 were injected with Se on day 0 . On days 0 ( $4 \mathrm{~h}$ following PBS or Se injection), 1 and 2, the test compound or vehicle was injected in each group subcutaneously ( $\mathrm{n}=6$ or 8 per group). Se, selenite; Hes, hesperetin; Hes-S, hesperetin stearic acid ester; Hes-O, hesperetin oleic acid ester; S.C., subcutaneously.

Switzerland). The levels of lens GSH were calculated by subtracting total GSH concentration from two times the concentration of oxidative GSH.

Measurement of AsA. Levels of AsA were determined using DCPIP as described previously (6). Lenses were homogenized in 0.1 M PBS ( $\mathrm{pH} 7.4$ ) and de-proteinized by using metaphosphoric acid. The lens homogenate was centrifuged to remove the proteins. The supernatant was titrated with DCPIP. Absorbance at $540 \mathrm{~nm}$ was measured in a microplate reader, Infinite M1000 (Tecan Ltd.).

Chaperone activity measurement. Chaperone activity was measured according to methods described previously, with minor modifications (13). Briefly, water-soluble lens proteins were mixed with aldehyde dehydrogenase (ALDH) in $50 \mathrm{mM}$ sodium phosphate buffer containing $100 \mathrm{mM} \mathrm{NaCl}$ (pH 7.0). ALDH aggregation was induced with 1,10-phenanthroline at $42^{\circ} \mathrm{C}$. Protein aggregation was monitored by measurement of light scattering at $360 \mathrm{~nm}$ using an Infinite M200PRO microplate reader (Tecan Ltd.).

Statistical analysis. All data are reported as means \pm standard error. Statistical analysis of data was performed using one-way analysis of variance with a post-hoc Tukey's multiple comparison test. SPSS version 24 software (IBM Corp., Armonk, NY, USA) was used for analysis. $\mathrm{P}<0.05$ was considered to indicate a statistically significant difference.

\section{Results}

Cataract classification. Thirteen-day-old SD rats were randomized into two groups and injected with either PBS (control groups: G1-G6) or Se (cataract groups: G7-G12), and each group was divided into five subgroups to further examine the effects of Hes, Hes-S, and Hes-O (Table I). Hes-S and Hes-O were synthesized as previously reported (10), and administered $4 \mathrm{~h}$ prior to injecting the rats with either PBS or Se, and then once daily for two days (total of three days). Six days after the PBS or Se injection, cataract classifications were determined as previously described (11). Fig. 2A-F show nuclear cataracts in rats from groups 7 to 12 , respectively (Fig. 2A-F). More than $80 \%$ of lenses from G7 (Se-treatment only) had mature grade 6 nuclear cataracts, with grade 5 cataracts present in the lenses of the remaining rats in that group (Fig. 2G). All lenses from control groups were transparent, and all had grade 1 cataracts (data not shown). The lenses of rats in G8, G11, and G12 (Se-Hes, Se-Hes-S, or Se-Hes-O co-treatment, respectively) lacked central opacity and/or had lower-grade cataracts compared to those of rats in groups G7, G9, or G10 (Se treatment only, Se-stearic acid, or Se-oleic acid co-treatment, respectively). In G8, 8, 8, 33, 33 , and $17 \%$ of rat lenses had cataract grades $5,4,3,2$, and 1 , respectively. In contrast, $8,8,42,25$, and $17 \%$ of rat lenses from G11 had cataract grades 5, 4, 3, 2, and 1, and 17, 58, $17 \%$, and 8 lenses in G12 had cataract grades $4,3,2$, and 1, respectively (Fig. 2G). These data suggest that treatment of the lens with hesperetin or hesperetin-derived compounds can delay Se-induced onset of cataracts.

GSH and AsA levels in the lens and plasma of Se-treated rats. The GSH and AsA levels in the lens and plasma were determined to evaluate the effects of hesperetin derivatives on the levels of antioxidant compounds in the lens. In the control groups (G1-G6), GSH levels in the lens showed no change either with or without treatment with hesperetin-derived compounds. In the G7 lenses, GSH levels were significantly decreased compared to the levels observed in control G1 lenses $(1.40 \mu \mathrm{mol} /$ wet weight vs. $0.41 \mu \mathrm{mol} /$ wet weight $)$ (Fig. 3A). Hesperetin treatment of rats with Se-induced cataracts rats prevented a reduction in GSH levels. The concentration of GSH in the lenses of rats in G8 lens was $0.98 \mu \mathrm{mol} /$ wet weight. Interestingly, the lens GSH levels in G11 or G12 rats were higher than those in G8. GSH concentrations in the lenses of rats in G11 were $1.04 \mu \mathrm{mol} /$ wet weight and in G12 were $1.2 \mu \mathrm{mol} /$ wet weight (Fig. 3A). Next, we measured the AsA concentrations in the lenses of rats with selenite-induced cataracts. In the control groups, AsA levels did not change regardless of treatment (G1-6). In the lenses of rats with Se-induced cataracts (G7), As A levels were significantly lower than those in the lenses of rats in the control group. The concentrations were $13.70 \mu \mathrm{g} / \mathrm{lens}$ wet weight in G7, and $32.53 \mu \mathrm{g} /$ wet weight in G1 (Fig. 3B). Co-treatment of lenses with Hes and $\mathrm{Se}(\mathrm{G} 8)$ prevented the reduction in AsA levels (24.12 $\mu \mathrm{g} /$ wet weight). AsA concentrations were higher in G11 and G12, those observed in in G7 rat lenses. AsA levels in G11 were $27.20 \mu \mathrm{g} /$ wet weight and $27.55 \mu \mathrm{g} /$ wet weight in G12.

Subsequently, we quantified the levels of plasma antioxidant compounds in the lenses. We did not observe any changes in the levels of GSH or AsA concentrations in the plasma following treatment with Hes-S or Hes-O, regardless of whether the lenses had cataracts or not (were transparent) 
A

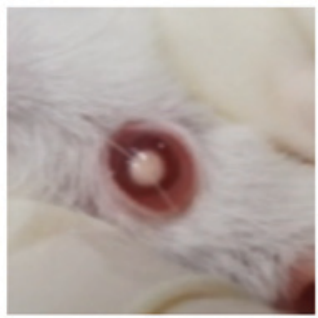

$\mathrm{D}$

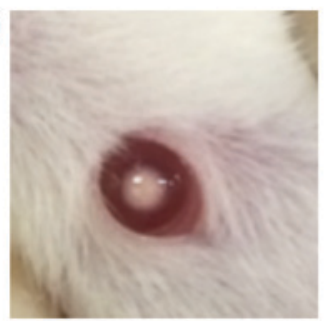

B

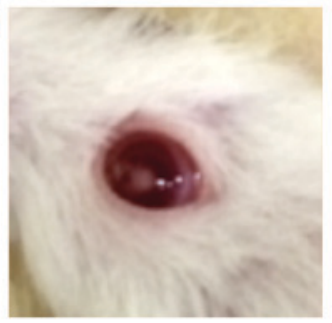

$\mathrm{E}$

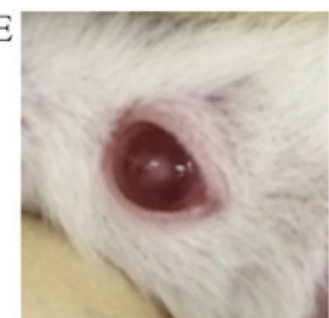

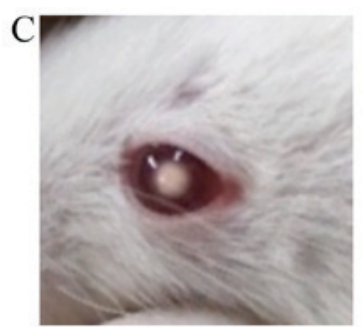

$\mathrm{F}$

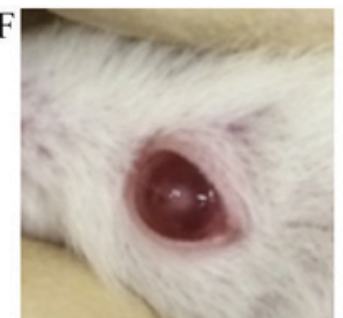

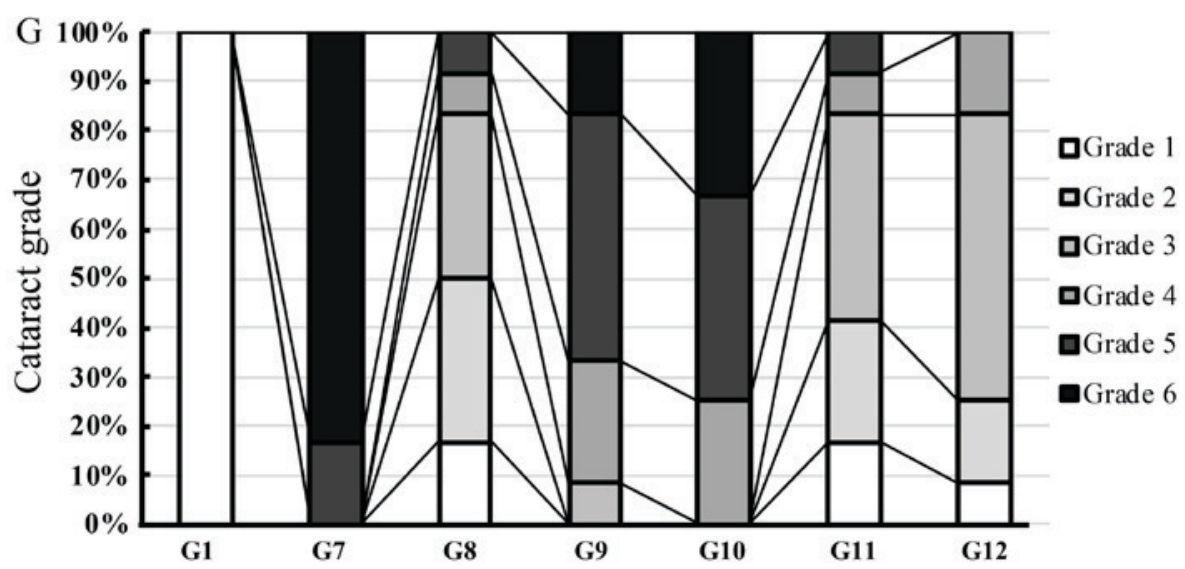

Figure 2. Anti-cataract effects of hesperetin and hesperetin derivatives. (A-F) Lenses from rats with selenite-induced cataracts: Lenses from a rat from (A) the Se-treated group (group 7: Cataract grade 6), (B) Se-Hes treatment group (group 8: Cataract grade 2), (C) Se-oleic acid treatment group (group 9: Cataract grade 5), (D) Se-stearic acid treatment group (group 10: Cataract grade 6), (E) Se-Hes-S treatment group (group 11: Cataract grade 2) or (F) Se-Hes-O treatment group (group 12: Cataract grade 2). (G) Cataract grade was determined for lenses from rats in the groups of rats that were treated with either hesperetin or hesperetin derivatives ( $n=6$ or 8 per group). G1, control group; G7-12, groups 7-12; Se, selenite; Hes, hesperetin; Hes-S, hesperetin stearic acid ester; Hes-O, hesperetin oleic acid ester.

(Fig. 4A and B). These results suggest that Hes-S and Hes-O prevent the reduction of antioxidants exclusively in the cataract lens, with no effect on systemic antioxidant levels.

Lens chaperone activity in cataract rats. The lens possesses chaperoning ability that helps to prevent protein aggregation and cataract formation. Cataract lenses are known to have weaker chaperone activity than normal, transparent lenses. Therefore, we measured the effect of both hesperetin and hesperetin-derived compounds on chaperone activity in cataract lenses. Chaperone activity was evaluated by the measurement of the time course of aldehyde dehydrogenase (ALDH) light scattering using 1,10-phenanthroline at $360 \mathrm{~nm}$ (Fig. 5A). The sample in ALDH alone did not show any light scattering (Fig. 5A, curve 9) and a mixture of ALDH and 1,10-phenanthroline in the absence of lens proteins demonstrated the largest amount of light scattering (Fig. 5A, curve 1). Lens proteins from mature cataracts in G7 were not able to suppress light scattering (Fig. 5A, curve 7). However, the lens proteins from Se-induced cataracts in rats treated with either Hes, Hes-S, or Hes-O showed reduced ALDH light scattering
(Fig. 5A, curve 5, 11, or 12, respectively). A mixture of ALDH, 1,10-phenanthroline, and lens proteins from transparent, normal lenses from G1 rats showed the lowest amount of ALDH light scattering (Fig. 5A, curve 8).

Light scattering in the absence of lens proteins was defined as $100 \%$ scattering. Suppression of ALDH light scattering was represented as relative $\Delta \mathrm{A} 360$, as indicated in the bar graph in Fig. 5B. The lens proteins from G1 suppressed ALDH light scattering, and those from G7 did not affect ALDH light scattering (Fig. 5B). The lens proteins from G8 protected against an increase in ALDH light scatter. However, light scattering suppression ability in G11 or G12 lens proteins was significantly stronger than that of G8 lens proteins. Light scattering reflected ALDH protein aggregation and the suppression of this aggregation was assumed to be dependent upon chaperone activity.

\section{Discussion}

As age-related cataract progression in humans is very slow and therapeutics must be applied for a long period of time to 

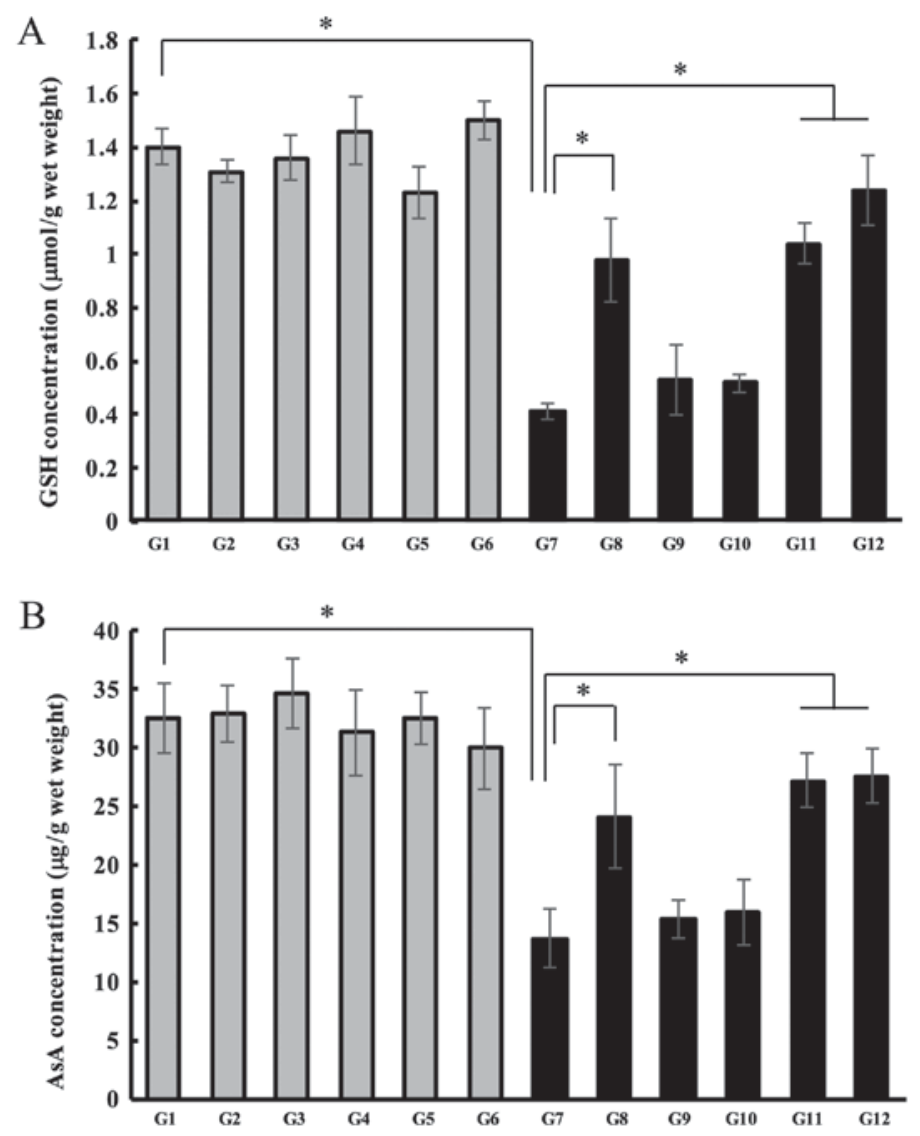

Figure 3. Effect of Hesperetin and hesperetin derivatives on the levels of lens antioxidant compounds in Se-induced cataracts. (A) GSH levels in lenses with Se-induced cataracts. (B) AsA levels in lenses with Se-induced cataracts. All results are expressed as the mean \pm standard error ( $\mathrm{n}=6$ or 8 per group). ${ }^{*} \mathrm{P}<0.05$, as indicated. G, group; Se, selenite; GSH, glutathione; AsA, ascorbic acid.
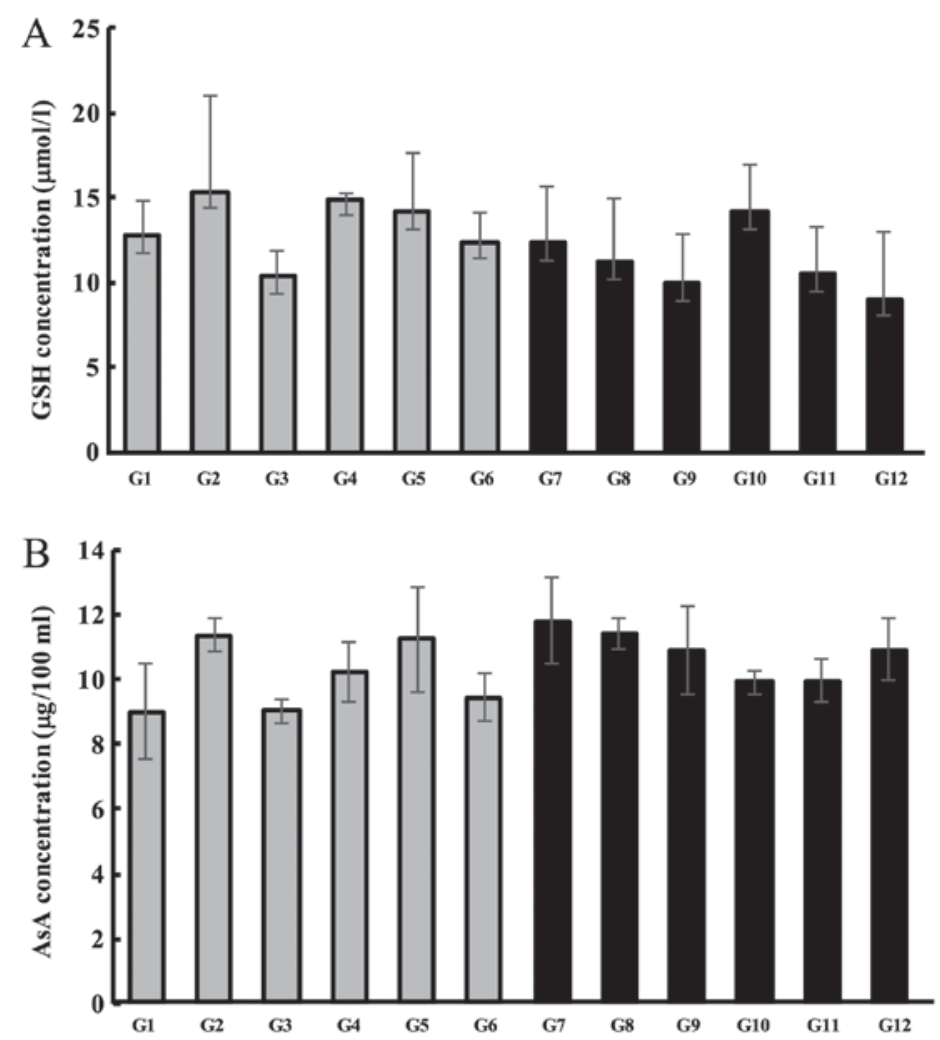

Figure 4. Effect of Hesperetin and hesperetin derivatives on the levels of plasma antioxidant compounds in Se-induced cataract. (A) Plasma GSH levels with Se-induced cataracts. (B) Plasma As A levels in rats with Se-induced cataracts. All results are expressed as the mean \pm standard error ( $\mathrm{n}=6$ or 8 per group). G, group; Se, selenite; GSH, glutathione; AsA, ascorbic acid. 

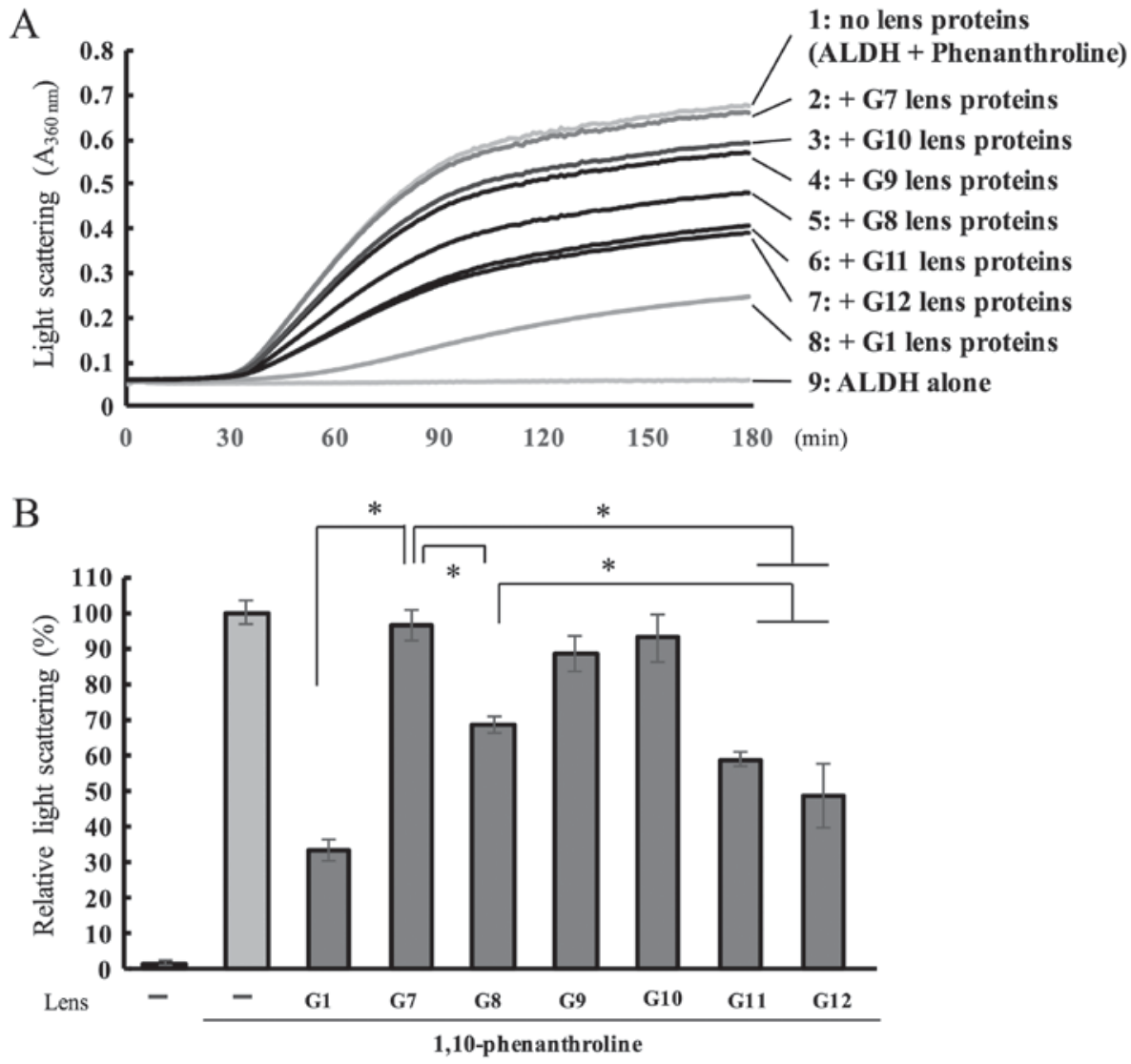

Figure 5. Effect of hesperetin and hesperetin derivatives on lens chaperone activity. (A) The time course of light scattering of ALDH at $360 \mathrm{~nm}$. Curve 1 represents the light scattering of the ALDH and 1,10-phenanthroline mixture without lens proteins. Curves 2-8 represent the light scattering of a mixture of ALDH and lens proteins from each group (from G7, G10, G9, G8, G11, G12 and G1, respectively). Curve 10 is that of ALDH alone. (B) Relative chaperone activity of cataract lens proteins with antioxidants was calculated using ALDH light scattering at 180 min following the addition of 1,10-phenanthroline. The change of light scattering of ALDH in the absence of water-soluble lens proteins was defined as $100 \%$. Bars represent the mean \pm standard error $(\mathrm{n}=6$ or 8 per group). "P<0.05, as indicated. G, group; ALDH, aldehyde dehydrogenase.

effectively ameliorate or prevent the development of cataracts, it is vital to study the long-term safety of pharmacological therapies for cataracts. Furthermore, identifying and developing effective anti-cataract agents in the human diet that can be consumed daily would provide significant health economic benefits.

In this study, we used an Se-induced cataract model that is well-characterized for screening potential anti-cataract agents. The selenite-induced cataract animal model can be efficiently induced by the administration of sodium selenite to rat pups younger than 16 days old. Cataracts appear within 3-5 days following sodium selenite administration. We previously reported that a 3 day course of administration of antioxidant compounds can ameliorate selenite-induced cataracts $(11,13)$. Manikandan et al (14) previously reported that curcumin injected $24 \mathrm{~h}$ before the administration of selenite could prevent the onset of cataract. Furthermore, Aydemir et al (15) reported that the administration of ebselen for 4 days inhibited oxidative stress in the lens and prevented selenite-induced cataract development in rats.

Hesperetin, a natural flavonoid isolated from orange rinds, has a flavanone backbone structure and is known to have strong antioxidant activity (16). As humans are unable to synthesize hesperetin, it is usually acquired from oranges and other orange-colored fruits. Hesperetin exhibits antioxidant activity by regulating the expression of antioxidant enzymes such as catalase, GSH peroxides, and GSH reductase $(17,18)$. Consumption of antioxidant compounds and maintaining a constant redox state in the lens are currently the recommended methods for preserving lens transparency, as cataracts are mainly caused by oxidative stress. We synthesized two hesperetin derivatives, Hes-S and Hes-O, that have a fatty acid linked to the 7-hydroxy position of hesperetin, to evaluate the effect of hesperetin derivatives on cataract onset by measuring cataract development, antioxidant levels, and lens chaperone activity.

In this report, we show that fatty acid-linked hesperetins have greater anti-cataract effects compared to that of the original chemical compound, hesperetin, especially for lens chaperone activity. We hypothesize that hesperetin fatty acid ester compounds may improve hesperetin pharmacokinetics due to the following effects: i) Improvement of uptake from subcutaneous areas into the bloodstream and systemically; ii) greater permeability across the blood-aqueous barrier; iii) hydrolysis of Hes-S or Hes-O into hesperetin by esterase(s) localized in the lens; and iv) trapping of hesperetin within the lens due to poor water solubility. Firstly, cellular uptake of Hes-S or Hes-O is thought to be enhanced by improving the lipophilicity of hesperetin which was achieved by linking it to either stearic acid or oleic acid. It is well known that drugs are generally absorbed by passive diffusion into the systemic 
circulation and that improving the lipophilicity of the drug can increase absorption rate. Furthermore, the absorption of lipophilic compounds is thought to be mediated primarily by membrane diffusion, whereas hydrophilic compounds appear to be absorbed via passive diffusion through intercellular junction pores $(19,20)$.

In this current study, we were unable to detect any differences in cataract classification or levels of antioxidant compounds in the between the hesperetin treatment and hesperetin derivative treatment groups. Indeed, neither hesperetin nor its derived compounds could be detected in the serum or lens by HPLC $4 \mathrm{~h}$ after injection (data not shown). However, we did observe significant differences in the retention of chaperone activity, we hypothesized that tiny amounts of hesperetin or its derivatives could reach the lens. It will be necessary to use a detector and/or HPLC system with a greater sensitivity to detect the presence of these compounds, and further studies will be needed to decipher how hesperetin and its derivatives reach the lens and/or interact with $\alpha$-crystallin at a molecular level to maintain lens chaperone activity.

The results of the current study indicate that hesperetin and hesperetin-derived compounds may delay or prevent cataract onset by preserving chaperone activity in lens proteins, and fatty acid-linked hesperetins had greater chaperone activity than that of the original compound (hesperetin). We previously reported that hesperetin treatment could prevent chaperone activity decreasing in the lens by preventing oxidative modification of $\alpha$-crystallin and retaining water solubility (9). Generally, $\alpha$-crystallin purified from the lens is used to measure chaperone activity. However, the water solubility of this protein and its concentration in the water-soluble fraction was changed after cataract onset. Therefore, we used measurements of total water-soluble protein to determine the lens chaperone activity.

Hes-O-treated rats displayed lower cataract grades, higher lens GSH levels, and stronger chaperone activity than Hes-S-treated rats. Although the carbon number of stearic acid is the same as that of oleic acid, but oleic acid has a cis-double bond in its structure. This double bond structure may affect the membrane permeability and pharmacokinetics of the hesperetin esters. Interestingly, cataract stages and markers of cataract development were improved in the animals treated with fatty acid (groups G9 and G10). We hypothesize that this improvement may be due to enhanced lens membrane stability. Further in-depth investigation is required to assess the pharmacokinetics of these compounds. In addition, further studies are needed to determine how Hes-S and Hes-O interact with lens proteins and prevent cataract onset at the molecular level, and how they affect the molecular disposition of antioxidants in vivo.

In addition to the anti-cataract effect, hesperetin has several general health benefits, such as anti-inflammatory properties, antihypertensive effects, and the improvement of very low-density lipoprotein (VLDL) metabolic abnormalities (21-23). We speculate that these health benefits may be more pronounced in Hes-S or His-O compared to that in the original compound (hesperetin).

As age-related cataract progression in humans is very slow and treatments must be applied for long periods of time to delay or prevent the development of cataracts, it is vital to study the long-term safety of pharmacological therapies.
As anti-cataract pharmaceutical therapies require long-term treatment, identifying affordable anti-cataract compounds that can be found ubiquitously in the human diet and that have no adverse effects is of paramount importance.

\section{Acknowledgements}

MP would like to thank the Graduate School of SIGMA Clermont, France, for the opportunity to be a visiting research student (2017) under an agreement between SIGMA Clermont and Keio University.

\section{Funding}

The present study was supported by grants from the Japan Society for the Promotion of Science KAKENHI (grant no. 16K18957), and from the Ministry of Education, Culture, Sports, Science, and Technology (MEXT)-supported program for Strategic Research Foundation at Private Universities (grant no. S1101003).

\section{Availability of data and materials}

The analyzed datasets generated during the study are available from the corresponding author on reasonable request.

\section{Authors' contributions}

YN and HT conceived and designed the present study. YN, MFT, TS and HT designed the methods. YN, MP, KF and NN performed the laboratory experiments. YN, NN and HT analyzed and interpreted the data. $\mathrm{YN}$ was major contributor in the writing of the manuscript.

\section{Ethics approval and consent to participate}

Keio University Animal Research Committee (Tokyo, Japan) approved all of the animal procedures that were performed in the present study [11014-(4)].

\section{Consent for publication}

Not applicable.

\section{Competing interests}

The authors declare that they have no competing interests.

\section{References}

1. Khairallah M, Kahloun R, Bourne R, Limburg H, Flaxman SR, Jonas JB, Keeffe J, Leasher J, Naidoo K, Pesudovs K, et al: Number of people blind or visually impaired by cataract worldwide and in world regions, 1990 to 2010. Invest Ophthalmol Vis Sci 56: 6762-6769, 2015.

2. Liu YC, Wilkins M, Kim T, Malyugin B and Mehta JS: Cataract. Lancet 390: 600-612, 2017.

3. Bergamini CM, Gambetti S, Dondi A and Cervellati C: Oxygen, reactive oxygen species and tissue damage. Curr Pharm Des 10: 1611-1626, 2004.

4. Pau H, Graf P and Sies H: Glutathione levels in human lens: Regional distribution in different forms of cataract. Exp Eye Res 50: 17-20, 1990. 
5. Kamei A: Glutathione levels of the human crystalline lens in aging and its antioxidant effect against the oxidation of lens proteins. Biol Pharm Bull 16: 870-875, 1993.

6. Nakazawa Y, Oka M, Bando M,Inoue T and Takehana M: The role of ascorbic acid transporter in the lens of streptozotocin-induced diabetic rat. Biomed Prev Nutr 1: 43-48, 2011.

7. Grey AC, Demarais NJ, West BJ and Donaldson PJ: A quantitative map of glutathione in the aging human lens. Int J Mass Spectrom (In press).

8. Nakazawa Y, Oka M, Bando M and Takehana M: Hesperetin prevents selenite-induced cataract in rats. Mol Vis 21: 804-810, 2015.

9. Nakazawa Y, Oka M, Tamura H and Takehana M: Effect of hesperetin on chaperone activity in selenite-induced cataract. Open Med (Wars) 11: 183-189, 2016.

10. Jeong TS, Kim EE, Lee CH, Oh JH, Moon SS, Lee WS, Oh GT, Lee S and Bok SH: Hypocholesterolemic activity of hesperetin derivatives. Bioorg Med Chem Lett 13: 2663-2665, 2003.

11. Ishimori N, Oguchi J, Nakazawa Y, Kobata K, Funakoshi-Tago M and Tamura $\mathrm{H}$ : Roasting enhances the anti-cataract effect of coffee beans: Ameliorating Selenite-induced cataracts in rats. Curr Eye Res 42: 864-870, 2017.

12. Sedlak J and Lindsay RH: Estimation of total, protein-bound and nonprotein sulfhydryl groups in tissue with Ellman's reagent. Anal Biochem 25: 192-205, 1968.

13. Nakazawa $\mathrm{Y}$, Nagai $\mathbf{N}$, Ishimori $\mathbf{N}$, Oguchi $\mathbf{J}$ and Tamura $\mathrm{H}$ : Administration of antioxidant compounds affects the lens chaperone activity and prevents the onset of cataracts. Biomed Pharmacother 95: 137-143, 2017.

14. Manikandan R, Thiagarajan R, Beulaja S, Sudhandiran G and Arumugam M: Effect of curcumin on selenite-induced cataractogenesis in Wistar rat pups. Curr Eye Res 35: 122-129, 2010.

15. Aydemir O, Güler M, Kaya MK, Deniz N and Üstündağ B: Protective effects of ebselen on sodium-selenite-induced experimental cataract in rats. J Cataract Refract Surg 38: 2160-2166, 2012.
16. Hwang SL and Yen GC: Effect of hesperetin against oxidative stress via ER- and TrkA-mediated actions in PC12 cells. J Agric Food Chem 59: 5779-5785, 2011.

17. St-Pierre J, Drori S, Uldry M, Silvaggi JM, Rhee J, Jäger S, Handschin C, Zheng K, Lin J, Yang W, et al: Suppression of reactive oxygen species and neurodegeneration by the PGC-1 transcriptional coactivators. Cell 127: 397-408, 2006.

18. Lu X, Kambe F, Cao X, Kozaki Y, Kaji T, Ishii T and Seo H: 3beta-Hydroxysteroid-delta24 reductase is a hydrogen peroxide scavenger, protecting cells from oxidative stress-induced apoptosis. Endocrinology 149: 3267-3273, 2008.

19. Effros RM and Mason GR: Measurements of pulmonary epithelial permeability in vivo. Am Rev Respir Dis 127: S59-S65, 1983.

20. Schneeberger EE, Airway and alveolar epithelia cell junctions. In: The lung: Scientific foundations. Crystal RG, West JB, Barnes PJ, Cherniack NS and Weibel ER (ed.) Raven Press, New York, pp205-214, 1991.

21. Ohtsuki K, Abe A, Mitsuzuwi H, Kondo M, Uemura K, Iwasaki Y and Kondo Y: Effects of long-term administration of hesperidin and glucosyl hesperidin to spontaneously hypertensive rats. J Nutr Sci Vitaminol (Tokyo) 48: 420-422, 2002.

22. Miwa Y, Mitsuzumi H, Sunayama T, Yamada M, Okada K, Kubota M, Chaen H, Mishima Y and Kibata M: Glucosyl hesperidin lowers serum triglyceride level in hypertriglyceridemic subjects through the improvement of very low-density lipoprotein metabolic abnormality. J Nutr Sci Vitaminol (Tokyo) 51: 460-470, 2005

23. Parhiz H, Roohbakhsh A, Soltani F, Rezaee R and Iranshahi M: Antioxidant and anti-inflammatory properties of the citrus flavonoids hesperidin and hesperetin: An updated review of their molecular mechanisms and experimental models. Phytother Res 29: 323-331, 2015. 